\title{
Pulse oximetry: an important first step in improving health outcomes, but is of little use if there is no oxygen
}

\author{
Peter P Moschovis, ${ }^{1}$ Patricia L Hibberd ${ }^{2}$
}

According to the most recent Unicef estimates, 5.9 million children under age 5 died in $2015 .^{1}$ In low-income countries, mortality in early childhood is dominated by prematurity, sepsis, and pneumoniadiseases that frequently result in hypoxemic respiratory failure. Since the introduction of pulse oximetry into clinical practice in the 1980s, pulse oximeters have become ubiquitous in high-income countries and are increasingly used in the diagnosis and management of childhood disease in middle and low-resource settings. There is intuitive appeal of a point-of-care device such as pulse oximetry to detect hypoxaemia, particularly in first-level healthcare facilities staffed by minimally trained health workers. However, it is prudent to examine the evidence regarding its use in clinical management and effect on outcomes, particularly in childhood pneumonia.

In this month's issue, Enoch et $a l^{2}$ evaluate the evidence underlying the assertion that pulse oximeters make a meaningful difference in the management of children. Five studies met criteria for inclusion in their systematic review, all before/after studies. Only one of these was conducted in a low-income country. Because of the heterogeneity in study design and outcomes, the authors were unable to conduct a meta-analysis but instead qualitatively summarised the findings.

Duke $e t a l^{3}$ conducted the only study that examined the effect of introducing pulse oximetry and oxygen concentrators on pneumonia-related mortality in Papua New Guinea. Improving the detection of hypoxaemia and treating it did reduce pneumonia mortality by $35 \%$. Based on their reported absolute risk reduction of

\footnotetext{
${ }^{1}$ Divisions of Global Health and Pulmonary/Critical Care Medicine, Massachusetts General Hospital, Boston, Massachusetts, USA; ${ }^{2}$ Division of Global Health, Massachusetts General Hospital for Children, Boston, Massachusetts, USA

Correspondence to Dr Peter P Moschovis, Divisions of Global Health and Pulmonary/Critical Care Medicine, Massachusetts General Hospital, 125 Nashua St. 8th Floor, Boston, MA 02114, USA; pmoschovis@mgh. harvard.edu
}

$1.75 \%$, the number of children needed to test with pulse oximetry and treat with supplemental oxygen in order to save one life (number needed to treat) was 57, a game-changing intervention in a disease that kills nearly one million young children per year. Notably, a portion of the observed reduction in mortality may also be related to the improved overall level of care children receive in a quality improvement initiative.

Every year, there are 1.5 million episodes of hypoxemic pneumonia requiring hospitalisation worldwide, ${ }^{4}$ and even though oxygen is on the WHO's list of essential medicines, patients in most district hospitals and many referral hospitals in sub-Saharan African and Asia are unlikely to receive oxygen because of the continued lack of a consistent supply of supplemental oxygen. ${ }^{5}$ It is difficult to imagine that the introduction of pulse oximetry alone into this context will improve pneumonia outcomes.

Several studies in the systematic review found that knowledge of a patient's oxygen saturation was associated with a change in clinical management, and in some cases, intensification of care (admission to a hospital, administration of oxygen). In other cases, pulse oximetry findings were reassuring and led providers to deintensify care. These findings are intuitive and not altogether surprising, but they demonstrate that physicians find the information generated by pulse oximetry useful in managing their patients.

Floyd et $a l^{6}$ recently developed a model to evaluate the benefit of pulse oximetry in reducing childhood pneumonia mortality in resource-poor settings. According to their analysis, combining pulse oximetry with implementation of WHO (integrated management of childhood illness) treatment guidelines would prevent 148000 pneumonia-related deaths annually in the 15 highest-burden countries, at a cost of less than US\$53 per disability-adjusted life year. Notably, the authors have found that at least $60 \%$ of hospitals must have oxygen available to their patients in order for pulse oximetry to offer any mortality benefit compared with IMCI-based clinical assessment alone. Unfortunately, in most resource-poor settings, oxygen availability is much less than $60 \%$.

The studies reviewed by Enoch et al, along with the results of Floyd's model, demonstrate that an improvement in the outcomes of children with hypoxaemia is possible, and that pulse oximetry plays a critical role in identifying children with the greatest need of oxygen. For the families of children with prematurity, sepsis and pneumonia, this is good news indeed, but to reap the benefit, we must find a way to ensure that when hypoxaemia is detected by pulse oximetry, there is a consistent supply of oxygen to treat the hypoxaemia.

Contributors PPM and PLH contributed equally to this editorial.

Funding PPM is supported by NIH/NHLBI grant 5 F32HL124951, and PLH is supported by NIH/NCCIH grant 5K24AT003683.

Competing interests None declared.

Provenance and peer review Commissioned; internally peer reviewed.

To cite Moschovis PP, Hibberd PL. Arch Dis Child 2016;101:685

Received 11 February 2016

Revised 15 March 2016

Accepted 19 March 2016

Published Online First 12 April 2016

SLinked

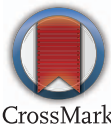

http://dx.doi.org/10.1136/archdischild-2015-309638

Arch Dis Child 2016;101:685.

doi:10.1136/archdischild-2015-310055

\section{REFERENCES}

1 You D, Hug L, Ejdemyr S, et al. Global, regional, and national levels and trends in under-5 mortality between 1990 and 2015, with scenario-based projections to 2030: a systematic analysis by the UN Inter-agency Group for Child Mortality Estimation. Lancet 2015;386:2275-86

2 Enoch AJ, English M, Shepperd S. Does pulse oximeter use impact health outcomes? A systematic review. Arch Dis Child 2016;101:694-700.

3 Duke $T$, Wandi $F$, Jonathan $M$, et al. Improved oxygen systems for childhood pneumonia: a multihospital effectiveness study in Papua New Guinea. Lancet 2008;372:1328-33.

4 Nair H, Simões EA, Rudan I, et al. Global and regional burden of hospital admissions for severe acute lower respiratory infections in young children in 2010: a systematic analysis. Lancet 2013:381:1380-90.

5 Manasyan A, Saleem S, Koso-Thomas M, et al. Assessment of obstetric and neonatal health services in developing country health facilities. Am J Perinatol 2013;30:787-94.

6 Floyd J, Wu L, Hay Burgess D, et al. Evaluating the impact of pulse oximetry on childhood pneumonia mortality in resource-poor settings. Nature 2015;528:S53-9. 